\title{
Die Unabhängigkeit der Dritten Gewalt im rechtsstaatlichen Strafrecht
}

- Bedingungen fortschreitender Marginalisierung - ${ }^{*}$

Die Notwendigkeit einer unabhängigen Dritten Gewalt im rechtsstaatlichen Strafrecht folgt aus grundlegenden theoretischen Überlegungen zu Staat und Strafrecht. Dabei kennzeichnet das rechtsstaatliche Strafrecht die strikte Rückbindung an die Staatstheorie, aus der sich die Pflicht zur Begrenzung staatlicher Strafmacht ergibt, sowie die Anerkennung des Menschen als mit eigenen Rechten ausgestattetes freies Individuum. Es ist also der Freiheitsschutzgedanke, der die Grenzen für die Wirkungen des Strafrechts markiert. Dies gilt es in einem ersten Schritt in den Blick zu nehmen, bevor im zweiten die Marginalisierung der Unabhängigkeit der Dritten Gewalt als Folge eines sich stetig weiterentwickelnden Präventionsstrafrechts umrissen werden soll.

\section{I. Überlegungen zum rechtsstaatlichen Strafrecht}

\section{Die Notwendigkeit einer absoluten Straftheorie zur Begründung des rechtsstaatlichen Strafrechts}

Die Idee eines rechtsstaatlichen Strafrechts ist zwingend verbunden mit der Idee des Rechtsstaats schlechthin. Die theoretische Fundierung basiert auf dem Rechtsstaatsmodell des deutschen Idealismus, insbesondere der Rechtsphilosophie Immanuel Kants. ${ }^{1}$ Die menschliche Vernunft ist es, die den Bürger dazu veranlasst, den Naturzustand hinter sich zu lassen, und sich im Staat zu vereinigen. ${ }^{2}$ Die Bedingungen der Vergesellschaftung sind die individuelle Freiheit, die Gleichheit und die Autonomie aller Bürger, also die Fähigkeit als eigene Rechtspersönlichkeit wahrgenommen und akzeptiert zu werden. ${ }^{3}$ Den Organisationsrahmen dieser Vergesellschaftung liefert die Idee des Gesellschaftsvertrages, wonach die einzelnen Bürger auf den Einsatz von Macht verzichten und diese Macht an den Staat überleiten, dem nunmehr das Gewaltmonopol zukommt. ${ }^{4}$ Dem Strafrecht vorgelagert sind die Idee der Gerechtigkeit sowie die Akzeptanz und Einhaltung der beschriebenen Konstitutionsbedingungen des Staates. Diese

* Der Beitrag basiert auf der Monographie des Verfassers: „Die Marginalisierung der Unabhängigkeit der Dritten Gewalt im System des Strafrechts", erschienen in den Frankfurter kriminalwissenschaftlichen Studien, Band 126, Peter Lang-Verlag, Frankfurt am Main 2010. Er stellt eine ergänzte und um Fußnoten erweiterte Fassung des Disputationsvortrages vom 31.5.2010 dar.

1 Vgl. zum Konstrukt einer ,absoluten Rechtsstaatlichkeit“ Fornauf, Die Marginalisierung der Unabhängigkeit der Dritten Gewalt im System des Strafrechts, 2010, 23 ff.

2 Kant, Metaphysik der Sitten, 1793, AA VI, 312; vgl. hierzu auch Kersting, Wohlgeordnete Freiheit, 1984, 210; Sinner, Der Vertragsgedanke im Strafprozeßrecht, 1999, 44 f.

3 Kersting (Fn. 2), 210, 239; Sinner (Fn. 2), 37; Harzer, Der Naturzustand als Denkfigur moderner praktischer Vernunft, 1994, $90 \mathrm{f}$.

4 Kersting (Fn. 2), 231. 
sind die Wegweiser des rechtsstaatlichen Strafrechts. Das Strafrecht dient in dieser Konzeption und in diesem Verständnis von Rechtsstaat als Mittel zur Begrenzung staatlicher Strafgewalt. ${ }^{5}$ Diese Perspektive - nämlich abgeleitete Macht vom Bürger zum Staat - beeinflusst die theoretische Legitimation des Strafrechts entscheidend: Aus ihr folgt eine ,staatsrechtliche Strafrechtstheorie“, ${ }^{6}$ die auf die Begrenzung des Strafrechts zielt, um so die individuellen Rechte des Einzelnen gegen den Staat zu schützen. Der Freiheitsschutzgedanke ist die ,juristische Sperre“ für „strafökonomische und kriminalpolitische" Absichten, ${ }^{7}$ kriminalpolitische folgenorientierte Zielsetzungen sind dem rechtsstaatlichen Begrenzungsstrafrecht fremd.

In seiner theoretischen Begründung muss das rechtsstaatliche Strafrecht auf einer absoluten Straftheorie beruhen: Nur so kann das formale Prinzip der Gleichheit zur Geltung gebracht werden, nur so kann eine der Gerechtigkeit entsprechende Strafe verhängt werden und nur so wird die Würde und die individuelle Freiheit jedes Bürgers angemessen berücksichtigt. ${ }^{8}$ Das Kennzeichen dieser absoluten strafrechtstheoretischen Begründung ist ein striktes Tatstrafrecht, also der Rückbezug auf die vergangene Rechtsverletzung, das verwirklichte zurechenbare Unrecht. Dieser Tatbezug liefert einen festen Maßstab zur Bemessung und Begrenzung staatlicher Strafgewalt. Nur hierdurch kommt die Wechselseitigkeit von Unrechtsverwirklichung und staatlicher Sanktionierung zum Ausdruck. Ein weiteres Kennzeichen ist die Vorhersehbarkeit und Bestimmbarkeit staatlicher Strafgewalt. Fundiert werden diese durch die Idee einer strikten Strafgesetzlichkeit (,nulla poena sine lege“), damit verbunden der Bestimmtheitsgrundsatz, das Analogieverbot, das Verbot rückwirkender Anwendung von Strafgesetzen und das Verbot von Gewohnheitsrecht. ${ }^{9}$ Diese Strafgesetzlichkeit ist es, die den Begrenzungscharakter des rechtsstaatlichen Strafrechts deutlich hervorhebt, indem sie die genaue Fixierung sanktionierender Rechtsverletzungen und staatlicher Strafverfolgungsverfahren im Blick hat. Sie richtet sich so gegen den Staat, legitimiert, kontrolliert und begrenzt ihn gleichermaßen sowohl bei der Festlegung strafrechtlich relevanten Unrechts als auch bei der Verfolgung der einzelnen Tat.

Es zeigt sich ein in sich geschlossenes Legitimationsmodell des rechtsstaatlichen Strafrechts. Sein Ziel ist die Begrenzung staatlicher Gewalt. Es dient dem Gedanken der Gerechtigkeit durch einen strikten Tatbezug und achtet so die dem Staat zugrunde liegenden Konstitutionsbedingungen, indem es die Würde und Freiheit jedes - machtabgebenden - Individuums achtet und gleichsam vor dem Machtmonopol des Staates schützt.

5 Naucke, Die Kriminalpolitik des Marburger Programms 1882, ZStW 94 (1982), 564.

6 Naucke, ,Schulenstreit“?, in: Festschrift für Winfried Hassemer, 2010, 565.

7 Naucke (Fn. 6), 565.

8 Fornauf (Fn. 1), 36 ff. m. w. N.

$9 \mathrm{Zu}$ den einzelnen Ausprägung der Strafgesetzlichkeit Naucke, Strafrecht - Eine Einführung, 10. Aufl. 2002, § 2. 


\section{Die Untauglichkeit relativer Straftheorien zur Begründung des rechtsstaatlichen Strafrechts}

Ein Präventionsstrafrecht und ein damit verbundener Legitimationsversuch rechtsstaatlichen Strafrechts durch eine relative Straftheorie kann diese Leistung keinesfalls erbringen. Das ist in der Konzeption des rechtsstaatlichen Strafrechts und des Rechtsstaats schlechthin ausgeschlossen. Den relativen Theorien fehlt zunächst ein strikter Tatbezug und damit ein fester Maßstab zur Bemessung und Begrenzung staatlicher Strafe. Die relativen Theorien fragen auch nicht danach, sondern vielmehr danach, welche Zwecke mit dem Strafrecht zu erreichen sind. Eine etwaige Begrenzung staatlicher Macht interessiert hierbei nicht, weil „die zweckmäßige Kriminalpolitik, je nach Staatspolitik, nur eine geringe oder gar keine Grenze verträgt." ${ }^{10}$ Die Zwecke indes sind flexibel, können von Fall zu Fall, von Situation zu Situation, von Zeit zu Zeit unterschiedlich sein und sind vor allem für kriminalpolitische Interpretationen offen. In all diesem zeigt sich die Unbestimmbarkeit und Unvorhersehbarkeit einer Präventionsstrafe, sie kann daher gar nicht den Anspruch einer an der Idee der Gerechtigkeit ausgerichteten Strafe erfüllen, weil jede staatliche Reaktion möglich wird. Das ausschließliche Abstellen auf einen staatlich zu verfolgenden Zweck durch die Bestrafung verkennt zudem die rechtsstaatliche und aus der Kantischen Tradition notwendig folgende Prämisse, den individuellen Bürger nicht als Zweck zu missbrauchen. Ihr liegt damit nicht nur ein anderes Rechtsstaatsbild zugrunde, sondern auch ein anderes Menschenbild.

Insgesamt folgt aus den präventiven Theorien ein Perspektivenwechsel: Es wird nicht mehr danach gefragt, was das Strafrecht darf, um die individuelle Freiheit der Bürger nicht über Gebühr zu beeinträchtigen, sondern was das Strafrecht kann, um die staatlichen Ziele - ohne Berücksichtigung der individuellen Freiheit - zu erreichen. Die Frage der Begrenzung des Strafrechts wird hierbei ,vernachlässigt“ und „von der Kriminalpolitik eingefangen und neutralisiert". ${ }^{11}$

\section{Das Schuldprinzip und der Grundsatz der materiellen Wahrheit als materielle Grundpfeiler des rechtsstaatlichen Strafrechts}

Maßgebliche materielle Kriterien rechtsstaatlichen Strafrechts im einzelnen Verfahren sind das Schuldprinzip und der Grundsatz materieller Wahrheit. Denn nur auf der Grundlage von Wahrheit und individueller, nachgewiesener Schuld kann strafrechtlicher Zugriff gerechtfertigt sein.

Das Schuldprinzip stellt das Maßprinzip eines rechtsstaatlichen Strafrechts dar, um so staatlichen Zugriff auf den Einzelnen zu legitimieren und $\mathrm{zu}$ begrenzen. ${ }^{12}$ Dem rechtsstaatlichen Strafrechtsmodell ist es wegen seiner Begrenzungsfunktion von vornherein immanent; normativ abzuleiten ist es aus dem Zusammenspiel des Rechtsstaatsprinzips sowie der Art. 1 und 2 Abs. 1 GG. ${ }^{13}$ Es versteht sich gleichsam als Erweiterung

10 Naucke (Fn. 6), 567.

11 Naucke (Fn. 6), 566.

12 Fornauf(Fn. 1), 55 ff. m. w. N.

13 Vgl. BVerfGE 20, 323 ff.; 57, 250 ff.; 86, 288 ff.; zuletzt ausdrücklich hervorgehoben im Urteil des Bundesverfassungsgerichts zum Vertrag von Lissabon BVerfGE 123, $267 \mathrm{ff}$. 
der Idee der Strafgesetzlichkeit (,nulla poena sine lege“), indem es diese um die subjektive Komponente der individuellen Vorwerfbarkeit ergänzt (,nulla poena sine culpa"). ${ }^{14}$ Hierin spiegelt sich zudem die notwendige Korrelation mit einer absoluten Straftheorie wider, weil sich die Bemessung der Strafe streng nach der zurückliegenden Tatbegehung richtet, die den Maßstab für eben jene zu verhängende Strafe liefert. ${ }^{15}$

Der Grundsatz der materiellen Wahrheit dient als prozessualer Umsetzungsmechanismus materieller Rechtsstaatlichkeit, weil eine dem Schuldgrundsatz entsprechende und damit gerechte Strafe nur gefunden werden kann, wenn der richterlichen Entscheidung der wahre Sachverhalt zugrunde gelegt wird. Das Ziel materieller Wahrheitssuche ergibt sich aus dem Ziel des rechtsstaatlichen Strafrechts, nämlich der Gerechtigkeit: Eine Strafe kann nur dann gerecht sein, wenn die normative Zurechnung individueller Schuld auf dem tatsächlich Geschehenen beruht. ${ }^{16}$ Dabei findet der Grundsatz nicht etwa nur in $\S 244$ Abs. 2 StPO seinen Niederschlag, sondern genießt gleichsam Verfassungsrang. ${ }^{17}$

Beide Grundsätze dienen letztlich der Gewährleistung von Gleichheit und Gerechtigkeit im Strafrecht. Sie stellen die materiellen Grundpfeiler des rechtsstaatlichen Strafrechts dar - und das auf verfassungsrechtlich gesichertem Fundament!

\section{Die Unabhängigkeit der Dritten Gewalt als formelles Programm von Rechtsstaatlichkeit}

Der Dritten Gewalt muss bei der Bewertung dieser materiellen Kriterien die entscheidende Rolle zukommen, denn nur sie kann - eben wegen ihrer Unabhängigkeit - effektiver Garant für die Umsetzung rechtsstaatlicher Gleichheit und Gerechtigkeit sein. Die strikte Bindung an Recht und Gesetz und die Befreiung von externen Einflüssen sind die Kennzeichen dieser Unabhängigkeit und damit die Fortführung der Strafgesetzlichkeit ins Verfahren. ${ }^{18}$ Nur so kann die entsprechende Kontrolle und Begrenzung staatlicher Machtausübung ausgeführt und durchgesetzt werden. Richterliche Unabhängigkeit ist damit die unabdingbare Voraussetzung eines rechtsstaatlichen Strafrechts und gleichsam Hauptforderung jeder Rechtsstaatlichkeit. Zusammenfassend bildet sie die formelle Seite der Durchsetzung materieller Rechtsstaatlichkeit.

Die Unabhängigkeit der Dritten Gewalt folgt dabei nicht nur notwendig aus den theoretischen Überlegungen zu einem rechtsstaatlichen Strafrecht, sondern auch aus verfassungsrechtlichen Vorgaben. ${ }^{19}$ Das Grundgesetz liefert in Bezug auf die richterliche Unabhängigkeit flankierende Leitlinien: Im Wesentlichen sind dies Art. 97, Art. 92, Art. 101 sowie Art. 20 GG. Die verfassungsrechtlichen Vorschriften lassen sich ihrem

14 Vgl. Naucke (Fn. 9), § 7, Rdn. 25, 27.

15 Vgl. Albrecht, Vom Präventionsstaat zur Sicherheitsgesellschaft, in: Festschrift für Winfried Hassemer, 2010, 3.

16 Stamp, Die Wahrheit im Strafverfahren, 1998, 27; Sternberg-Lieben, Einstellungsurteil oder Freispruch, ZStW 108 (1996), 727, Fn. 31; vgl. auch Neumann, Materiale und prozedurale Gerechtigkeit im Strafverfahren, ZStW 101 (1989), 52, dort Fn. 1.

17 Vgl. BVerfGE 57, 250 ff.; 63, 46 ff.; 70, $297 \mathrm{ff}$.

18 Fornauf (Fn. 1), 294; ähnlich Naucke, Konturen eines nach-präventiven Strafrechts, KritV 1999, 344.

19 Fornauf(Fn. 1), 65 ff. 
Gehalt nach in objektive und subjektive Bedingungen formeller Rechtsstaatlichkeit einteilen. Dabei versteht sich - auf objektiver Seite - der Grundsatz der Gewaltenteilung als institutionelle Absicherung eines rechtsstaatlichen Verfahrens, indem dieser einerseits die Gewalten organisatorisch trennt, aber andererseits durch die gleichzeitige Verschränkung der Gewalten diese an Recht und Gesetz bindet. Auf subjektiver Seite steht dem Beschuldigten im Strafrecht ein aus den Art. 101, 92 GG resultierender Anspruch auf ein neutrales Verfahren sowie auf einen gesetzlich vorgesehenen, unabhängigen Richter zu. Zur Gewährleistung dieses Anspruchs ist es staatliche Verpflichtung, sowohl dem einzelnen Richter als auch der Dritten Gewalt als Institution die notwendigen Mittel zur Verfügung zu stellen, damit der Schutz individueller Interessen in ausreichendem Maße gewährleistet werden kann. Die Notwendigkeit einer unabhängigen Dritten Gewalt ergibt sich demnach nicht nur aus einem theoretischen Konstrukt, sondern auch aus zwingenden verfassungsrechtlichen Vorgaben.

Erst die Korrelation von materieller Rechtsstaatlichkeit, die ihren Ausdruck im Schuldprinzip und im Grundsatz materieller Wahrheit findet, und formeller Rechtsstaatlichkeit, also die Gewährleistung eines dem Beschuldigten gegenüber neutralen Verfahrens - gesichert und kontrolliert durch die Unabhängigkeit der Dritten Gewalt -, lässt die Forderung nach Gleichheit und Gerechtigkeit nicht nur als programmatische Hoffnung erscheinen, sondern als realistisches und umsetzbares Ziel des rechtsstaatlichen Strafrechts.

\section{Die Marginalisierung der Unabhängigkeit der Dritten Gewalt im rechtsstaatlichen Strafrecht}

Die moderne Strafrechtsentwicklung steht - entgegen dem Charakter eines rechtsstaatlichen Strafrechts als retrospektives Begrenzungsstrafrecht - unter der Prämisse prospektiver Prävention. ${ }^{20}$ Hierbei sind insbesondere zwei Entwicklungslinien auszumachen, die das Gesamtbild moderner Strafrechtsentwicklung prägen und die der Unabhängigkeit der Dritten Gewalt zunehmend ihre Bedeutung nehmen. Dabei geht es einerseits um die Schwerpunktverlagerung im Strafverfahren zugunsten des Ermittlungsverfahrens und den damit verbundenen Bedeutungszuwachs exekutiver Ermittlungsbehörden, andererseits um die Konsensorientierung im modernen Präventionsstrafrecht. Beide Entwicklungen bedrohen nicht nur das rechtsstaatliche Strafrecht, sondern auch die Unabhängigkeit der Dritten Gewalt nachhaltig.

\section{Die Schwerpunktverlagerung des Strafverfahrens und die Folgen für die Unabhängigkeit der Dritten Gewalt}

Das rechtsstaatliche Strafrecht kennzeichnet eine Ausbalancierung der am Strafverfahren beteiligten Organe und der einzelnen Verfahrensabschnitte. Dieses Konzept ist die Umsetzung eines der tragenden rechtsstaatlichen Prinzipien, nämlich der Gewaltentei-

20 Vgl. nur Albrecht, Prävention als problematische Zielbestimmung im Kriminaljustizsystem, KritV 1986, 54 ff.; ders., Das Strafrecht auf dem Weg vom liberalen Rechtsstaat zum sozialen Interventionsstaat, KritV 1988, 182 ff.; Naucke (Fn. 18), 336 ff. 
lung. Man spricht von strafprozessualer Gewaltenteilung ${ }^{21}$ : Das Gericht kontrolliert die Exekutive, das streng förmliche Hauptverfahren kontrolliert das exekutivisch dominierte Ermittlungsverfahren. Innerhalb des Ermittlungsverfahrens kommt dem Richtervorbehalt wesentliche Bedeutung zu. Zwar ist die Staatsanwaltschaft als Exekutivbehörde „Herrin des Ermittlungsverfahrens“"22, denn ihr kommt die Aufgabe zu, bei Vorliegen eines hinreichenden Tatverdachts den zugrundeliegenden Sachverhalt zu erforschen und letztlich über die Erhebung einer Anklage zu entscheiden. Sie unterliegt bei ihren Ermittlungen allerdings den Begrenzungen durch Richtervorbehalte und damit der Kontrolle durch die Dritte Gewalt. Beim Institut des Richtervorbehaltes zeigt sich das Anliegen des rechtsstaatlichen Strafrechts besonders deutlich: Es geht darum, exekutiven Ermittlungsmaßnahmen die Unabhängigkeit der Dritten Gewalt entgegenzusetzen, um so staatlichen Zugriff zu kontrollieren und gleichsam zu begrenzen. ${ }^{23}$

Dabei lässt sich diese Funktion nicht nur theoretisch ableiten, sondern sie ergibt sich auch aus historischer und teleologischer Sicht. ${ }^{24}$ Historisch gewachsen ist der Richtervorbehalt wegen eines Misstrauens gegenüber der Exekutive. Obsolet wird dieses Misstrauen durch die auch für die Exekutive geltende strenge Bindung an Recht und Gesetz (Art. 20 Abs. 3 GG) keineswegs, weil auch das Bundesverfassungsgericht einer richterlichen Entscheidung höhere Legitimation beimisst ${ }^{25}$ und die verfassungsrechtlich nach wie vor geltenden Richtervorbehalte für Freiheitsentzug (Art. 104 Abs. 2 GG) sowie Wohnungsdurchsuchungen und Wohnraumüberwachungen (Art. 13 Abs. 2 bis 5 GG) immer noch Bestand haben. Teleologisch gesehen ist der Richtervorbehalt zudem Ausdruck gegenseitiger Gewaltenkontrolle im Strafverfahren und erfüllt zugleich für den Beschuldigten eine präventive Rechtsschutzfunktion (Art. 19 Abs. 4 GG). Zusammenfassend kommt dem Institut des Richtervorbehaltes im Ermittlungsverfahren daher eine fundamentale Bedeutung zu, wenn es darum geht, die subjektiven Rechte des Beschuldigten gegenüber exekutiver Dominanz zu stärken.

Die moderne Strafrechtsentwicklung ebnet indes den Weg für einen klaren Bedeutungsverlust dieser prozeduralen Grundrechtsabsicherung, dessen hauptsächlicher Grund in einer politischen Missachtung liegen dürfte, weil die Normierung von Richtervorbehalten im einfachen Recht optional und flexibel wird. Er beruht aber auch auf strukturellen Defiziten, weil die Möglichkeit zur Überprüfung der Rechtmäßigkeit einer Maßnahme dem Gericht normativ und faktisch entzogen wird, sowie auf exekutiven Umgehungsmechanismen, die sich aus der eigentlich subsidiären Anordnung der Maßnahme wegen Gefahr in Verzug sowie aus dem allgemeinen Trend zur Geheimhaltung des Ermittlungsverfahrens ergeben. Nicht zuletzt scheint die Schwächung des Richtervorbehaltes aber auch eine Mitursache bei der Dritten Gewalt selbst zu haben, lässt sich doch aus sehr geringen Ablehnungsquoten und aus einer meist nur oberflächlichen Bearbeitung, in der eigene richterliche Abwägungen kaum Eingang in den Beschluss finden, ein gewisses Fehlverständnis über die Bedeutung des Richtervorbehaltes nicht

21 Hassemer, Grundlinien eines rechtsstaatlichen Strafverfahrens, KritV 1990, 271.

22 Beulke, Strafprozessrecht, 10. Aufl. 2008, Rdn. 79.

23 Vgl. Rabe von Kühlewein, Der Richtervorbehalt im Polizei- und Strafprozessrecht, 2001, $414 \mathrm{~m}$. w. N.

24 Fornauf(Fn. 1), $103 \mathrm{ff}$.

25 Vgl. BVerfGE 77, 1 (51). 
leugnen. ${ }^{26}$ Das Bundesverfassungsgericht hat diese Tendenzen zur Kenntnis genommen und versucht, die fundamentale Bedeutung des Richtervorbehaltes zu stärken, indem es rekurriert auf die grundrechtssichernde Funktion von Richtervorbehalten bei Gefahr im Verzug, ${ }^{27}$ auf die Notwendigkeit richterlicher Bereitschaftsdienste als organisatorische Voraussetzung von Richtervorbehalten, ${ }^{28}$ auf die Konkretisierung von Begründungsanforderungen im Rahmen von Richtervorbehalten, ${ }^{29}$ auf die Notwendigkeit der Unterrichtung des Beschuldigten bei verdeckten Ermittlungsmaßnahmen ${ }^{30}$ sowie auf die Ausweitung von Verwertungsverboten von Beweismitteln, die aufgrund rechtswidriger Maßnahmen erlangt wurden. ${ }^{31}$

Die Schwächung von Richtervorbehalten ist indes nur die notwendige Folge des modernen Präventionsstrafrechts, das den Schwerpunkt des Verfahrens immer weiter ins Ermittlungsverfahren vorverlagert. Zugleich verschieben sich die Gewichte innerhalb des Kriminaljustizsystems zugunsten der Strafverfolgungsbehörden. Die Folge ist nicht nur der beschriebene Bedeutungsverlust des Richtervorbehaltes, sondern gleichsam ein weitgehender genereller Verlust richterlicher Kontrolle über staatliche Ermittlungsmaßnahmen. ${ }^{32}$ Die Funktion prozeduraler Grundrechtsabsicherungen durch die unabhängige Dritte Gewalt gerät dabei in den Hintergrund.

Aus der verfahrensstrukturellen Verlagerung zu Lasten der richterlich geführten Hauptverhandlung sowie aus der damit einhergehenden Machtverschiebung zugunsten der Exekutive folgt ein allgemeiner und umfassender Bedeutungsverlust der Unabhängigkeit der Dritten Gewalt sowie ein gewandeltes Verständnis des Ermittlungsverfahrens. Dieses dient nun weniger der Vorbereitung einer förmlichen Hauptverhandlung als vielmehr einer interessengeleiteten Erledigung von Verdachtsfällen. Es zeichnet sich aus durch eine fehlende Trennschärfe zum Polizeirecht sowie durch flexible und kaum mehr kontrollierbare Ermittlungsmaßnahmen. ${ }^{33}$ Dies beeinflusst und marginalisiert die Rolle des Richters entscheidend: Das nunmehr auf Flexibilität, Schnelligkeit und Effizienz ausgerichtete Ermittlungsverfahren verdrängt die ausführliche Rechtskontrolle staatlicher Ermittlungsmaßnahmen durch den unabhängigen Richter. Ausgiebiger Grundrechtsschutz durch eine unabhängige Dritte Gewalt wird der (vermeintlichen) Effektivität der Strafverfolgung geopfert.

\section{Die Tendenz zur Konsensualisierung im Strafverfahren und die Folgen für die Unabhängigkeit der Dritten Gewalt}

Die Konsensorientierung betrifft das förmliche Strafverfahren insgesamt. Sie lässt sich exemplarisch an den Entwicklungen des Strafbefehlsverfahrens, der Einstellungsmöglichkeit des $\S 153$ a StPO, der informellen wie nunmehr formellen Verständigung im

26 Zum Ganzen Fornauf (Fn. 1), 118 ff.

27 BVerfGE 103, $142 \mathrm{ff}$.

28 BVerfGE 103, $142 \mathrm{ff} ; 105,239 \mathrm{ff}$.

29 BVerfGE 96, 44 ff.; 103, $142 \mathrm{ff}$.

30 BVerfGE 109, $279 \mathrm{ff}$.

31 BVerfG NJW 2005, 1917; NJW 2006, 2684.

32 Fornauf(Fn. 1), $263 \mathrm{ff}$.

33 Fornauf (Fn. 1), 266 f. 
Strafverfahren und der Kronzeugenregelung eindrucksvoll belegen. ${ }^{34}$ Wesentlicher Grund dürfte vor allem die Ausweitung des materiellen Strafrechts sein und die dadurch hervorgerufene Überforderung des Kriminaljustizsystems. ${ }^{35}$ Die Folgen dieser Entgrenzung des materiellen Strafrechts lassen sich nur dadurch kompensieren, dass sich die Praxis des Kriminaljustizsystems in Erledigungsstrategien flüchtet, die auf Effizienz und Flexibilität angelegt sind. Es werden Verfahrenserledigungsmodelle gesucht, die ihren Hauptnenner darin finden, dass sie in Bezug auf rechtsstaatliche Mindeststandards defizitär ausgestaltet sind und diese Defizite durch die Beteiligung des Beschuldigten zu kompensieren suchen, insofern also konsensorientiert sind. Kennzeichen dieser Verfahrensarten ist nicht mehr rechtsstaatliche Prinzipiengebundenheit, sondern die schnelle und einfache Abwicklung von Verdachtsfällen. Richterliche Schuldüberzeugung auf der Basis materieller Wahrheit ist dabei nicht mehr gefragt, weil sie für die gewünschte Abwicklung zu viele, vor allem zeitliche und förmliche Komplikationen aufweist. Dabei kommt die Konsensorientierung innerhalb des Systems des Strafrechts durch folgende Erledigungsmodelle zum Ausdruck, die als Produzenten der Marginalisierung der Unabhängigkeit der Dritten Gewalt jeweils kurz skizziert werden sollen.

a) Der Strafbefehl und die Einstellung unter Auflagen als konsensorientierte Verfahrenserledigungen

Das Strafbefehlsverfahren und seine derzeitige Ausgestaltung sind ausgerichtet auf einen zu großen Anwendungsbereich (Vergehen, die in die Zuständigkeit des Einzelrichters oder des Schöffengerichts fallen), einen zu breiten Sanktionsrahmen (Freiheitsstrafe bis zu einem Jahr auf Bewährung) sowie eine nur auf Verdacht beruhende Rechtsfolge ${ }^{36}$ deren Legitimation sich auf dem Konsens zwischen Staat und Beschuldigten gründet. ${ }^{37}$ Letzteres muss die notwendige Schlussfolgerung aus der gesetzlichen Systematik sowie aus der beabsichtigten Praktikabilität sein. Denn wäre die richterliche Schuldfeststellung die Grundlage eines Strafbefehls, würde dies die Frage aufwerfen, weshalb die Zustimmung des Beschuldigten im Nachhinein noch erforderlich ist. Dieses Zustimmungserfordernis kann daher nur bedeuten, dass sich die Legitimation eines Strafbefehls eben nicht aus richterlicher Schuldüberzeugung auf der Grundlage materieller Wahrheit ergibt, sondern aus einem Konsens der am Verfahren Beteiligten. Der gesuchte Konsens mit dem Beschuldigten soll die rechtsstaatlichen Defizite ausgleichen, zumal es sich innerhalb des Strafbefehlsverfahrens um vermeintlich nicht so gravierende Rechtsfolgen handelt. Das Strafbefehlsverfahren stellt sich somit einerseits als konsensorientiertes Verfahren dar, andererseits belegt es zugleich die notwendige Verbindung der Konsensorientierung mit der Verhängung einer bloßen Verdachtsstrafe. Denn die Suche nach Konsens ist nur dort geboten, wo die eigentliche Legitimation durch Schuldprinzip und materielle Wahrheit im rechtsstaatlichen Strafrecht keine Wirkung mehr entfaltet und auch nicht mehr gefragt ist.

34 Fornauf(Fn. 1), $144 \mathrm{ff}$.

35 Vgl. nur Fischer, Rechtsmißbrauch und Überforderung der Strafjustiz, NStZ 1997, 214 f.

36 Fornauf(Fn. 1), $156 \mathrm{ff}$.

37 Fornauf (Fn. 1), $159 \mathrm{ff}$. 
Das Strafbefehlsverfahren eignet sich durch seine Ausgestaltung daher zwar für die Abwicklung von Massen- und Bagatellkriminalität, es bereitet aber in rechtsstaatlicher Hinsicht erhebliche Probleme. Denn es wendet sich gegen notwendige Grundbedingungen des rechtsstaatlichen Strafrechts, nämlich

- gegen das Schuldprinzip und den Grundsatz der materiellen Wahrheit, weil die Legitimation staatlicher Sanktionen auf einem Konsens mit dem jeweiligen Beschuldigten beruht und nicht auf nachgewiesener individueller Schuld;

- gegen den Grundsatz strafprozessualer Gewaltenteilung, weil es die kontradiktorische Struktur des Strafverfahrens auflöst und durch die Ausrichtung auf ein Zusammenwirken von Judikative und Exekutive eine effektive gegenseitige Kontrolle aufgibt und

- gegen den Grundsatz des rechtlichen Gehörs, weil eine Anhörung des Beschuldigten vor Erlass eines Strafbefehls nicht vorgesehen ist.

Anknüpfungspunkte für konsensuale Elemente innerhalb der Strafprozessordnung bietet auch die Ausgestaltung des $\S 153$ a StPO als Möglichkeit der Einstellung unter Auflagen. Eingeführt $1974^{38}$ stellt diese keineswegs ein Verfahren der Opportunität dar, weil sie im Ergebnis für den Beschuldigten keine folgenlose Einstellung bedeutet. Vielmehr zeichnet sich das Verfahren im Wesentlichen durch die Zustimmung der Beteiligten aus. Der Beschuldigte muss dabei zunächst die Einstellung unter Auflagen akzeptieren und die entsprechenden Auflagen erfüllen. Erst durch seine Bereitschaft kann das Verfahren letztlich erledigt werden. Parallel zum Strafbefehlsverfahren geht es darum, von der Erhebung einer öffentlichen Klage abzusehen und das Verfahren anderweitig zu beenden. Voraussetzungen sind neben der Zustimmung des Beschuldigten, dass die verhängten Auflagen und Weisungen geeignet sind, das öffentliche Interesse an der Strafverfolgung zu beseitigen und dass die Schwere der Schuld einer Einstellung nicht entgegensteht. Ungeachtet der Problematik, dass die Staatsanwaltschaft als Exekutivbehörde Überlegungen zu Schuldgesichtspunkten anstellen soll, zeichnet sich auch $\S 153$ a StPO durch einen zu breiten Anwendungsbereich (Vergehen, bei denen die Schwere des Schuld nicht entgegensteht) und die Möglichkeit von Sanktionierungen ( $\$ 153$ a Abs. 1 S. 2 Nr. 1-6 StPO) aus. Insbesondere letzteres ist mit der Einbettung in die Opportunitätsvorschriften der Strafprozessordnung nicht in Einklang zu bringen. ${ }^{39}$

In seiner Ausgestaltung widerspricht $§ 153$ a StPO somit nicht nur den Vorgaben einer rechtsstaatlich verträglichen Opportunität im Sinne prozessualer Entkriminalisierung, sondern er löst als konsensuales Verfahren auch die kontradiktorische Struktur des Strafverfahrens auf. In seiner rechtsstaatlichen Bedenklichkeit richtet sich das Verfahren der Einstellung unter Auflagen insbesondere

- gegen den Bestimmtheitsgrundsatz des Art. 103 Abs. 2 GG und damit zugleich gegen den Gleichheitsgrundsatz des Art. 3 GG, weil $\S 153$ a StPO keine bestimmbaren gesetzlichen Voraussetzungen einer Verfahrenseinstellung erkennen lässt, diese vielmehr ins Ermessen der Exekutive stellt;

- gegen den Grundsatz strafprozessualer Gewaltenteilung, weil die Exekutive sowohl Kompetenzen der Judikative wahrnimmt, wenn sie strafrechtlich relevantes Verhalten

38 BT-Drucks. 7/550, 190, $297 \mathrm{ff}$.

39 Vgl. Fornauf (Fn. 1), 178 ff. 
mit Sanktionen ahnden darf, als auch der Legislative, wenn sie über die Voraussetzungen der Strafwürdigkeit von Rechtsverletzungen entscheidet sowie

- gegen das Schuldprinzip und den Grundsatz der materiellen Wahrheit, weil die verhängte Sanktion nicht mehr auf einer richterlichen Schuldüberzeugung beruht, sondern auf dem gefundenen Konsens aller Verfahrensbeteiligten.

Die der Strafprozessordnung bereits seit langem bekannten Verfahren des Strafbefehls und des $\S 153$ a StPO genießen nicht nur hohe praktische Relevanz, sondern ähneln sich in ihrer jeweiligen Ausgestaltung. Als konsensorientierte Verfahren entfernen sie sich von der kontradiktorischen Grundstruktur des Strafverfahrens, die das Prinzip der Gewaltenteilung aufgreift und durch die gegenseitige Kontrolle von Justizorganen und Verfahrensabschnitten zur Geltung bringt. Gemeinsam ist ihnen die rechtsstaatliche Bedenklichkeit im Hinblick auf das Schuldprinzip und den Grundsatz der materiellen Wahrheit. Dies ist aber nur die unumgängliche Folge der Konsensorientierung. Denn gerade die Einhaltung und Gewährleistung dieser beiden materiellen Fundamentalprinzipien soll durch die alternative Legitimationssuche nach einem Konsens mit dem Beschuldigten ersetzt werden.

Die beiden genannten Verfahrenserledigungsmodelle und deren Ausgestaltungen sind die Folgen eines materiell überlasteten Strafrechts: Diesem Strafrecht geht es nicht mehr in erster Linie um die Einhaltung rechtsstaatlicher Prinzipien, sondern um die effektive Erledigung sämtlicher Strafverfahren. Im Bereich der leichten und der mittleren Kriminalität scheint dies zu der - dem rechtsstaatlichen Strafrecht fremden - Gleichung zu führen: Je geringer der Tatvorwurf und je geringer die aufzuerlegenden Sanktionen, desto mehr muss der Beschuldigte auf den prinzipiellen Schutz seiner Rechte verzichten bzw. desto weniger muss der Staat auf die Einhaltung dieser Rechte achten, solange sich der Beschuldigte damit nur einverstanden erklärt. Dass diese Gleichung durch die Möglichkeit der Verfahrensverständigung nunmehr das gesamte Spektrum der Kriminalität betrifft, scheint angesichts der materiellen Überlastung und der defizitären Strukturen innerhalb des Kriminaljustizsystems, insbesondere innerhalb der Dritten Gewalt, unumgänglich und im Sinne einer effektiven Abwicklung auch politisch willkommen, wenn auch rechtsstaatlich höchst bedenklich.

Im Strafbefehlsverfahren und bei der Einstellungsentscheidung nach $\S 153$ a StPO sehen die Folgen der durch die Gemengelage materieller Überlastung und struktureller Defizite notwendig werdenden Orientierung auf den Konsens mit dem Beschuldigten für die Unabhängigkeit der Dritten Gewalt wie folgt aus: Die richterliche Entscheidung besteht nur noch in einem formalen, weil gesetzlich (noch) vorgesehenen Bestätigen des exekutivischen Sanktionierungsvorschlags. Dem Richter kommt allenfalls die Rolle eines Urkundsbeamten zu, der zwar formal die Legitimation einer vermeintlich materiell gerechtfertigten Strafe liefern, inhaltlich aber keinen Einfluss auf den Ausgang des Verfahrens und die Sanktionierung nehmen soll. ${ }^{40}$ Der unabhängige Richter wird so zum Büttel der Exekutive.

40 Näher Fornauf (Fn. 1), 271 ff., 279 ff. mit empirischen Belegen, die dieses Ergebnis zwar nicht definitiv belegen, es aber zumindest plausibel erscheinen lassen. 


\section{b) Verfahrensabsprachen}

Auch die informellen wie nunmehr die als „Verständigung im Strafverfahren“ in $\S 257$ c StPO geregelten Verfahrensabsprachen haben ihren Ausgangspunkt in der Konsensorientierung des Strafverfahrens. Im Gegensatz zu den bereits dargestellten Verfahrensweisen wird dabei allerdings nicht nur die Zustimmung des Beschuldigten zur aufzuerlegenden Sanktion gesucht. Die Verfahrensabsprache geht darüber hinaus, indem der Konsens mit den Beteiligten darüber gesucht wird, wie das gesamte Verfahren $\mathrm{zu}$ gestalten und letztlich $\mathrm{zu}$ beenden ist. Die Beendigung erfolgt schließlich formal durch ein richterliches Urteil, wobei dieses auf den durch die Beteiligten abgesprochenen Bedingungen und Umständen beruht. Der Idee nach geht es also um den konsentierten Abbruch des Wahrheitsfindungsprozesses. Der Eingriff in die kontradiktorische Struktur des Strafverfahrens liegt dabei auf der Hand: Im Vordergrund des Hauptverfahrens steht nicht mehr die richterlich angeleitete Suche nach materieller Wahrheit, sondern das ,gesamte Prozessergebnis [soll] lange vor dem Ende der Hauptverhandlung und außerhalb derselben durch die Zentralfiguren des Prozessgeschehens in Abwesenheit anderer punktgenau festgelegt ${ }^{\text {“41 }}$ werden.

Nach der Entscheidung des Großen Senats des $\mathrm{BGH}^{42}$ entschied sich nunmehr auch der Gesetzgeber, Stellung zu den Verfahrensabsprachen zu beziehen. Das gesetzgeberische Einschreiten gegen die durch die justizielle Praxis entwickelten informellen Verfahrensabsprachen ist aus rechtsstaatlicher Perspektive sicherlich zu begrüßen, weil demokratisch legitimierte und genau ausgestaltete Vorgaben für das Strafverfahren die notwendige Rechtssicherheit und Gleichheit in der Rechtsanwendung gewährleisten. Dabei darf der Appell des Großen Senats an den Gesetzgeber, „die Zulässigkeit, und bejahendenfalls, die wesentlichen rechtlichen Voraussetzungen und Begrenzungen von Urteilsabsprachen gesetzlich zu regeln" ${ }^{43}$ allerdings nicht so verstanden werden, als sei der Gesetzgeber lediglich aufgefordert gewesen, die bisher in der höchstrichterlichen Rechtsprechung entwickelten Grundsätze ${ }^{44}$ der Verfahrensabsprachen in Gesetzesform zu gießen. ${ }^{45}$ Auch ein striktes Verbot gesetzlich nicht vorgesehener Verfahrenserleichterungen und Verfahrenserledigungen wäre eine Alternative gewesen, die vor dem Hintergrund der ausgeführten rechtsstaatlichen Grundbedingungen weniger bedenklich, wenn nicht sogar rechtsstaatlich geboten gewesen wäre.

Der Gesetzgeber entschied sich bekanntermaßen anders und wollte konkrete Voraussetzungen schaffen, die das Verfahren, das Zustandekommen, den Inhalt und die Rechtsfolgen einer Verfahrensabsprache regeln, dabei aber „den Rahmen, den die Grundsätze des geltenden Strafprozessrechts ziehen“, nicht verändern. ${ }^{46}$ Dieses hehre und begrüßenswerte Ziel des Gesetzgebers musste indes einer Sisyphosaufgabe gleichen, weil der Verfahrensabsprache gerade ein konsensualer Charakter und eben nicht die kontradiktorische Struktur des traditionellen rechtsstaatlichen Strafverfahrens $\mathrm{zu}$ Grunde

41 Eschelbach, Absprachenpraxis versus Wiederaufnahme des Verfahrens, HRRS 2008, $191 \mathrm{~m}$. w. N.

42 BGHSt 50, $40 \mathrm{ff}$.

43 BGHSt 50, 40 (64).

44 Im Wesentlichen wohl vor allem BGHSt 43, $195 \mathrm{ff}$.; 45, $227 \mathrm{ff}$. sowie letztlich 50, $40 \mathrm{ff}$.

45 So jedenfalls muss die Formulierung ,bejahendenfalls“" gedeutet werden.

46 BT-Drucks. 16/13095, 2. 
liegt. ${ }^{47}$ Verfahrensabsprachen gewinnen ihre Attraktivität gerade aus der Informalität und dem damit verbundenen Beschleunigungseffekt, denn ,die Beseitigung von Förmlichkeiten ist geradezu der Witz der Absprache.“48 Dieser Aspekt ist es aber auch, was das legislative Einziehen formaler Vorgaben durch das Gesetz zur Regelung der Verständigung im Strafverfahren wirkungslos machen dürfte und was letztlich auch die im rechtsstaatlichen Strafrecht vorgesehene Kontrolle durch eine unabhängige Dritte Gewalt unmöglich machen wird.

Verfahrensabsprachen und rechtsstaatliches Strafrecht sind nicht kompatibel. Sie leben aus unterschiedlichen Überzeugungen und haben unterschiedliche Zielsetzungen. Verfahrensabsprachen werden sich nur dann in ein Strafprozessrechtsmodell integrieren lassen, wenn Schuldprinzip und der Grundsatz der materiellen Wahrheit aufgegeben und durch ein am Konsens orientiertes Legitimationsmodell ersetzt werden.

Das Verständigungsverfahren rückt aber nicht nur von Schuldprinzip und dem Grundsatz der materiellen Wahrheit ab, es rückt auch von der Vorstellung eines Hauptverfahrens ab, das kontradiktorisch ausgestaltet ist und durch einen unabhängigen Richter geleitet wird. Die Rolle des Richters als unabhängiger Entscheider wird nachhaltig beeinflusst. Zwar werden die Verfahren durch richterliches Urteil beendet, die Grundlage und Entscheidungsvoraussetzungen dieses Urteils unterliegen jedoch nicht mehr der unabhängigen richterlichen Verfahrensleitung, sondern dem - in Wahrheit ökonomisch motivierten ${ }^{49}$ - Konsens aller Verfahrensbeteiligten. Weil der Richter aktiv auf einen Konsens hinwirken soll, ist er gezwungen, seine unabhängige Verfahrensleitung aufzugeben..$^{50}$

Die Verständigung im Strafverfahren - gleich ob formell oder informell - wendet sich also insgesamt

- gegen den Grundsatz der materiellen Wahrheit, weil das Ziel des Strafverfahrens nicht mehr die Feststellung des tatsächlich Geschehenen ist, sondern vielmehr der Konsens über ein dem Urteil zugrunde zu legendes Geschehen - ähnlich der formellen Wahrheit im Zivilprozess;

- gegen das Schuldprinzip, weil einerseits die Schuld nicht an der materiellen, sondern nur an einer formellen Wahrheit gemessen wird und andererseits die Gefahr besteht, dass dem entgegenkommenden Beschuldigten ein Strafmaß auferlegt wird, das dem tatsächlichen Schuldvorwurf nicht mehr entspricht sowie

47 Ähnlich Saliger, Absprachen im Strafprozess an den Grenzen der Rechtsfortbildung, JuS 2006, 12 („Quadratur des Kreises“); Jahn/Müller, Der Widerspenstigen Zähmung - Aktuelle Gesetzgebungsvorschläge zu den Urteilsabsprachen im Strafprozess, JA 2006, 686 („einen Pudding an die Wand nageln").

48 Hassemer, Förmlichkeiten im Strafprozess, in: In dubio pro libertate, Festschrift für Klaus Volk, 2009, 221; Hettinger, Von der Gleichheit vor dem Gesetz zur Ungleichheit vor Gericht?, in: Festschrift für Egon Müller, 2008, 267.

49 Trüg, Lösungskonvergenzen trotz Systemdivergenzen im deutschen und US-amerikanischen Strafverfahren, 2002, 112.

50 Im Blick hat der Gesetzgeber dabei allerdings nur den hauptamtlichen professionellen Richter. Im Gesetzgebungsverfahren überhaupt nicht berücksichtigt wurden die Laienrichter, die regelmäßig an der Verfahrensabsprache nicht beteiligt sind. Dass auch sie durch ihre Unabhängigkeit zum Schutz des Beschuldigten und zu der Rechtsstaatlichkeit des Verfahrens beitragen, scheint dem Gesetzgeber entgangen zu sein. Vgl. hierzu Fischer in seiner Stellungnahme im Rechtsausschuss, Protokoll der 134. Sitzung des Rechtsausschusses, 57. 
- gegen den Grundsatz der strafprozessualen Gewaltenteilung, weil die Judikative in eine Rolle versetzt wird, in der sie aktiv an der Konsensfindung teilnehmen soll, und so die ihr zukommenden Aufgaben unabhängiger Rechtsprechung und effektiver Kontrolle staatlicher Strafverfolgung kaum mehr wahrnehmen kann.

Damit verändert sich zugleich das Ziel des Strafverfahrens: Nicht mehr der hoheitliche Richterspruch wird angestrebt, sondern der gemeinsam gefundene Konsens der Beteiligten. Das streitige Hauptverfahren und die richterliche Schuldüberzeugung werden überlagert von einer funktionalen Suche nach einem Konsens, mit dem alle leben können. Den Rollen- und Funktionswechsel des unabhängigen Richters vollendet dabei das Initiativrecht zur Verständigung, weil dadurch seine eigentliche Aufgabe zu denaturieren droht: Nicht mehr die Gewährleistung eines neutralen Verfahrens steht im Vordergrund, sondern die Verfolgung eigener Interessen, die sich vordergründig an der Prozessökonomie und nicht am Schutz des Beschuldigten orientieren dürften. Dabei scheint diese Entwicklung auch anzuknüpfen an die bereits festgestellte Schwerpunktverlagerung zu Lasten der richterlichen Hauptverhandlung. Denn angesichts des deutlichen Übergewichts der Exekutivbehörden und angesichts der Tendenz zur Geheimhaltung scheint der Hauptverhandlung nur noch eine bloße Bestätigungsfunktion zuzukommen. ${ }^{51}$ Die Weichen des Strafverfahrens werden früher gestellt, nämlich im Ermittlungsverfahren. Nach der richterlich geführten, förmlichen und prinzipienorientierten Hauptverhandlung besteht kein Verlangen mehr. Denn ,wozu aufwendige Gerichtsverfahren, wenn Staatsanwälte das im Vorfeld mit links erledigen“"52 können. Dass wegen der weitgehend exekutiven Vorbestimmung keine umfangreiche Wahrheitssuche mehr stattfinden muss, erscheint daher plausibel und letztlich nur konsequent, rechtsstaatlich aber keineswegs vertretbar.

c) Die allgemeine Kronzeugenregelung als Paradebeispiel eines grenzenlosen Präventionsstrafrechts

Die Marginalisierung der Unabhängigkeit der Dritten Gewalt findet nicht nur Produzenten im Strafverfahrensrecht, sondern auch im materiellen Recht. So dürfte die allgemeine Kronzeugenregelung ebenfalls zum Bedeutungsverlust der unabhängigen Dritten Gewalt beitragen. Mit dem neu eingefügten $\S 46$ b StGB verspricht sich der Gesetzgeber zwar Aufklärungs- und Präventionshilfe. ${ }^{53}$ Das gewählte Mittel ist allerdings seit jeher höchst umstritten, stellt es doch einen „Fremdkörper in unserem Verfahrensrecht ${ }^{\text {"54 }}$ dar. ${ }^{55}$ Die Neuregelung spiegelt indes den Wandel des Strafrechts von einem prinzipiengeleiteten Begrenzungsstrafrecht hin zu einem konsensorientierten Präventionsstrafrecht deutlich wider: Einerseits beruht sie auf einer Abwägung von Präventionsbedürfnis und strikter Prinzipienorientierung, die dem rechtsstaatlichen Strafrecht

51 Ähnlich Albrecht, Die Kriminalisierung der Dritten Gewalt, ZRP 2004, 261.

52 Albrecht (Fn. 51), 261.

53 BT-Drucks. 16/6268.

54 Hettinger, Entwicklungen im Strafrecht und Strafverfahrensrecht der Gegenwart, 1997, 49.

55 Mushoff, Die Renaissance der Kronzeugenregelung, KritV 2007, 366 ff. m. w. N. 
fremd ist. ${ }^{56}$ Andererseits stellt sie durch die kooperative Ausgestaltung eine „Spielart ${ }^{\prime 57}$ der Absprachenpraxis dar und ist damit ein weiterer Schritt in die Richtung konsensorientierter Prävention. Das Institut des Kronzeugen dient als Paradebeispiel eines „modernen“ Präventions- und Interventionsstrafrechts. ${ }^{58}$

Vor dem Hintergrund des rechtsstaatlichen Begrenzungsstrafrechts wendet sich die Kronzeugenregelung des $\S 46$ b StGB

- gegen die materiellen Grundlagen des rechtsstaatlichen Strafrechts, insbesondere gegen das Schuldprinzip, weil nicht mehr die begangene Tat Anknüpfungspunkt für eine strafrechtliche Sanktion ist, sondern der Wert des Beschuldigten im Hinblick auf weitere Aufklärungs- und Präventionshilfe;

- gegen den Gleichheitsgrundsatz, weil nicht jedem Beschuldigten die Möglichkeit einer Strafreduzierung geboten wird, sondern nur denjenigen, die Besonderes zu bieten haben sowie

- gegen die staatliche Strafverfolgungspflicht, weil sich der Staat vorbehält, in gewissen Konstellationen von der Strafverfolgung bzw. der Strafe ganz oder teilweise abzusehen.

Obwohl es sich bei der Kronzeugenregelung um eine Regelung im materiellen Recht handelt, besteht auch durch sie enormes Bedrohungspotential für die Unabhängigkeit der Dritten Gewalt. Dieses folgt hauptsächlich aus der Begrenzung des zeitlichen Anwendungsbereiches auf das Ermittlungsverfahren, wodurch zugleich die Schwerpunktverlagerung des Strafverfahrens nochmals deutlich zum Ausdruck kommt. Der Richter im Hauptverfahren findet nach Anwendung der Kronzeugenregelung folgende Situation vor: Er kann entweder den durch die Strafverfolgungsbehörden in Aussicht gestellten Strafnachlass bestätigen und führt so die vorgesehene strafrechtliche Gewaltenteilung ad absurdum, weil keine echte Kontrolle mehr besteht - sowohl innerhalb des Kriminaljustizsystems als auch innerhalb des Verfahrens. Oder er kann - weil die Strafzumessung seine originäre und alleinige Aufgabe ist - gegen den redewilligen Beschuldigten eine angemessene Strafe verhängen. Hierbei würde der Richter die Rechtssicherheit und den Vertrauensschutz des Beschuldigten gegenüber staatlichen Zusagen unterminieren und ebenfalls rechtsstaatliche Grundprinzipien in Frage stellen.

Es ist eine Pattsituation, die der Gesetzgeber dem rechtsanwenden Richter zumutet, weil dieser - gleich wie er entscheidet - dem rechtsstaatlichen Strafrecht schadet. Rein faktisch dürfte aber dem Richter kaum ein echter Entscheidungsspielraum verbleiben, weil er der Intention der Aufklärungs- und Präventionshilfe folgend regelmäßig die erste Variante wählen, also den exekutivischen Vorschlag bestätigen wird, weil das Entgegenkommen des Beschuldigten und seine Mitwirkung am Konsens gerade das ist, was verlangt wird und belohnt werden soll. Von der eigentlichen richterlichen Funktion, nämlich aus dem Inbegriff der Hauptverhandlung ein unabhängiges, angemessenes und letztlich gerechtes Urteil zu fällen, wird nach Anwendung der Kronzeugenregelung wenig übrigbleiben. Die Unabhängigkeit der Dritten Gewalt wird so nachhaltig ausgehebelt.

56 Hassemer, Unverfügbares im Strafprozess, in: Rechtsstaat und Menschenwürde, Festschrift für Werner Maihofer, 1988, $183 \mathrm{f}$.

57 MühlhofflPfeiffer, Der Kronzeuge, ZRP 2000, 125.

58 Salditt, Allgemeine Honorierung besonderer Aufklärungshilfe, StV 2009, 377. 


\section{Zusammenfassung}

Diese kurze Bestandsaufnahme belegt nicht nur das fortwährende Abschleifen traditionell rechtsstaatlicher Prinzipien, ${ }^{59}$ sondern skizziert zugleich wesentliche Produzenten der Marginalisierung der Unabhängigkeit der Dritten Gewalt im System des Strafrechts. Durch die Schwerpunktverlagerung im Strafverfahren ergibt sich ein der Idee strafprozessualer Gewaltenteilung fremdes Übergewicht der Exekutive zu Lasten des Einzelnen. Es zeigt sich zudem ein Perspektivenwechsel innerhalb des Ermittlungsverfahrens weg von einem grundrechtsschonenden Verbrechensbekämpfungsbegrenzungsrecht hin $\mathrm{zu}$ einem grundrechtsverzehrenden Verbrechensbekämpfungsrecht, der letztlich den Bedeutungsverlust des Richtervorbehaltes als Institut der Grundrechtsschutzes sowie die Marginalisierung der Unabhängigkeit der Dritten Gewalt vorantreibt. Die Konsensorientierung belastet und gefährdet die traditionellen Zielsetzungen des rechtsstaatlichen Strafprozesses. Die richterliche Schuldüberzeugung auf der Grundlage der materiellen Wahrheit ist nicht mehr das, was den „neuen“ Strafprozess auszeichnet. Vielmehr geht es diesem um Opportunität, Ökonomie und Effizienz. ${ }^{60}$ Der Gedanke des Konsenses folgt dabei einer Eigendynamik, die in der Ausweitung des materiellen Strafrechts und der daraus resultierenden Überlastung des Kriminaljustizsystems ihren Ausgangspunkt findet. Sowohl im Strafbefehlsverfahren als auch in der Einstellungsmöglichkeit des $\S 153$ a StPO zeigt sich dies. Beide Verfahren zeichneten sich ursprünglich gerade nicht durch eine Konsensorientierung aus, sondern einerseits durch ein verkürztes, summarisches Verfahren, das gleichwohl richterliche Schuldüberzeugung und materielle Wahrheit im Blick hatte (Strafbefehl), andererseits durch das Opportunitätsprinzip (§ 153 a StPO) ${ }^{61}$ Der Bezug zu einem etwaigen Konsensprinzip ergab sich vielmehr erst durch die stetige Ausweitung beider Verfahren - sowohl hinsichtlich des Anwendungsbereiches als auch der zu verhängenden Rechtsfolge.

Ähnliches gilt für die Verfahrensabsprachen, die - gesetzlich erst gar nicht vorgesehen - erst im Laufe der Zeit durch die Praxis entwickelt wurden, wobei deren Ursprung nicht nur in einer Überlastung des Kriminaljustizsystems lag, sondern deren Entwicklung auch maßgeblich durch die Strafverteidiger forciert worden sein dürfte. ${ }^{62}$ Gleichwohl nahm die Praxis die Möglichkeit informeller Absprachen an. Daran konnte auch die höchstrichterliche Rechtsprechung, daran wird vermutlich auch der neu eingeführte $\S 257 \mathrm{c} \mathrm{StPO}$ nichts ändern. ${ }^{63}$

Mit der Kronzeugenregelung scheint diese Eigendynamik ihren derzeitigen Höhepunkt erreicht zu haben. Sie ist der Prototyp eines ausufernden Präventionsstrafrechts und an rechtsstaatlicher Bedenklichkeit vorerst kaum zu übertreffen: Der öffentliche Strafanspruch wird zur Nebensache, wenn die dem Kronzeugen vorgeworfene Tat nur gravierend genug ist, ihn zur Aussage zu bewegen, um dadurch entweder Taten, in die er selbst verwickelt ist, oder aber auch ganz andere Taten schneller aufklären oder ver-

59 Zum Prinzipienverlust auch Hassemer, Strafrecht, Prävention, Vergeltung, ZIS 2006, 269.

60 Sinner (Fn. 2), 137.

61 Fornauf (Fn. 1), 203.

$62 \mathrm{Zu}$ den Entstehungsbedingungen Hettinger (Fn. 48), 267 ff. m. w. N.

63 Vgl. Hamm, Quo vadis Strafprozess?, in: Festschrift für Egon Müller, 2008, 248; Fischer, Regelung der Urteilsabsprache, NStZ 2007, 435; Hassemer (Fn. 48), 221; Hettinger (Fn. 48), 267. 
hindern zu können. Der Anspruch auf Durchsetzung von Recht und Gesetz in Bezug auf die dem Kronzeugen vorgeworfene Tat muss der Prävention weichen. Wo Rechtsfeststellung und Rechtsdurchsetzung aber nicht mehr gefragt sind, teilt die Unabhängigkeit der Dritten Gewalt deren Schicksal. Ungleichbehandlungen und Rechtsunsicherheit finden so Eingang in das Strafverfahren, Verfahrensgerechtigkeit bleibt hingegen auf der Strecke.

Das Gesamtbild dieser Entwicklung ist eindeutig: Das „moderne“ Strafrecht strebt nach effizienter Strafverfolgung und Straftatenbekämpfung sowie flexibler und schneller Erledigungsverfahren, die aus Sicht der Praxis auch noch bestenfalls einer unabhängigen Kontrolle zu entziehen sind. Obsolet werden dabei vor allem das Schuldprinzip und der Grundsatz der materiellen Wahrheit sowie die Schutzfunktion einer unabhängigen Dritten Gewalt. Dies kennzeichnet die Marginalisierung der Unabhängigkeit der Dritten Gewalt im System des Strafrechts, deren Wirkung für den Rechtsstaat leichtfertig verkannt wird. Das rechtsstaatliche Strafrecht, das als ein in sich geschlossenes Modell des Freiheitsschutzes dargestellt wurde, wird ersetzt durch eine opportune Prävention, die vom rechtsstaatlichen Modell einer unabhängigen Dritten Gewalt abfällt. Richterliche Entscheidungen verlieren ihre inhaltliche Legitimationskraft und damit ihre Bedeutung für den individuellen Freiheitsschutz und die Begrenzung staatlicher Strafmacht. Übrig bleibt nur eine formale Legitimationswirkung für staatlichen Machtzugriff, die inhaltliche Kontrollfunktion wird dagegen kupiert. Die Dritte Gewalt wird so funktionalisiert für ein effektives Konzept opportuner Prävention. ${ }^{64}$ Dem Rechtsstaat wird nachhaltig geschadet.

64 Ähnlich Albrecht, Die Bedrohung der Dritten Gewalt durch irrationale Sicherheitspolitik, DRiZ 1998, 329. 